\title{
Digestible Lysine Requirements of Broilers
}

\section{-Author(s)}

Bernal LEPI

Tavernari FC"

Rostagno HS

Albino LFT"

Universidade Federal de Viçosa

" Researcher at Embrapa Suínos e Aves Concórdia, SC, Brasil

III Professor of the Department of Animal Science-Federal University of Viçosa, Viçosa, MG, Brazil Avenida Peter Henry Rolfs, s/n. e-mail: lalbino@ufv.br

\section{Mail Adress}

Corresponding author e-mail address Luiz Fernando Teixeira Albino.

Department of Animal Science-Federal University of Viçosa, Viçosa, MG, Brazil Avenida Peter Henry Rolfs, s/n. E-mail: lalbino@ufv.br

\section{-Keywords}

Digestible lysine, requirement, performance, broiler.

\section{ABSTRACT}

Modern broilers have been submitted to continuous genetic improvement, and therefore, their nutritional requirements must be constantly updated to ensure their performance. Two experiments were carried out to evaluate different digestible lysine levels for starter (1021 days) and grower (22-35 days) phases. The experiments were carried out with male and female Cobb 500 broilers, distributed according to a randomized block experimental design in a $5 \times 2$ factorial arrangement (5 increasing digestible lysine levels $\times 2$ sexes), totaling 10 treatments, with 8 replicates of 22 and 20 birds during the starter and grower phase, respectively. Digestible lysine levels of 1.06, 1.12, 1.18, 1.24, and 1.30 were used in the starter diets (10-21 days) and 0.9, 0.98, $1.04,1.10$, and $1.16 \%$ in the grower diets (22-35 days). Based on the statistical analyses of the evaluated performance parameters, digestible lysine requirements for maximum performance were determined as $1.22 \%$ for males and $1.24 \%$ for females in the starter phase, and $1.16 \%$ for both sexes in the grower phase. Carcass and performance results indicate that digestible lysine requirements vary with sex and evaluated production parameter. Considering the most relevant broiler production parameters, in 22- to 35-d-old males, digestible lysine requirement for breast meat yield $(1.16 \%)$ was higher than those for feed conversion ratio $(1.07 \%)$ and weight gain $(1.05 \%)$.

\section{INTRODUCTION}

The accurate knowledge of the nutritional requirements of essential amino acids and of the nutritional composition of the feedstuffs is essential to correctly balance feeds, and therefore, nutritional recommendations must be continuously updated. Several research institutions in Brazil and abroad carry out experiments to determine poultry nutritional requirements; however, different values are reported as a function of differences in methodology, genetics, and environment.

According to Leclercq (1998b), one of the main difficulties in establishing amino acid requirement values is the criteria used to determine these requirements (weight gain, breast weight, or feed conversion ratio), statistical analyses, and the lack of knowledge on non-essential amino acids. Several studies use weight gain as the single criterion to determine amino acid requirements or amino acid ratios, but this may lead to an underestimation of the requirements of other parameters because weight gain requirements are usually lower than those for feed conversion ratio, breast yield and abdominal fat.

For a same weight gain, some amino acids, such as lysine, may reduce lipid deposition and increase protein accretion, as clearly demonstrated by Grisoni (1991) and Leclercq (1998a). Increasing lysine dietary concentration above the requirement for weight gain may result 
in higher breast yield. Therefore, lysine requirements are higher when breast yield is used instead of weight gain as the criterion to determine those requirements. Feed conversion ratio is also frequently utilized to estimate amino acid requirements, and consequently, to calculate ideal amino acid profile in dietary protein. According to Han \& Baker (1994), the requirements of several amino acids to optimize feed conversion are higher than those needed for weight gain.

The objective of the present study was to determine the nutritional requirements of digestible lysine based on the ideal protein concept of male and female Cobb 500 broilers during two rearing phases: starter (1021 days of age) and grower (22-35 days of age), and to evaluate the effects of increasing digestible lysine levels on the carcass traits of 35-d-old male and female Cobb 500 broilers.

\section{MATERIALS AND METHODS}

Two experiments were carried out at the Poultry Sector of the Department of Animal Science of the Federal University of Viçosa. The first was performed during the starter phase (10-21 days of age) and the second during the grower phase (22-35 days of age) of male and female COBB 500 broilers, separately reared.

Birds were housed in a conventional masonry broiler house and distributed in 80 pens $(1.0 \times 2.25$ $\mathrm{m})$. The house was $3 \mathrm{~m}$ high, and had fibrocement deck roof, concrete floor covered with wood-shavings litter, $0.40 \mathrm{~m}$ high side walls with wire mesh and plastic curtains to control temperature and drafts.

Infrared lamps were used for brooding. Birds were offered feed and water ad libitum during the entire experimental period. During the first week, water was supplied in chick drinkers, and feed in trays, after which tube feeders and nipple drinkers were used.

Maximum and minimum temperatures were monitored during each experiment using three maximum-minimum thermometers placed at different location inside the house at birds' height.

In the first experiment, 1760 male and female Cobb 500 broilers were used. A randomized block experimental design in a $5 \times 2$ factorial arrangement was applied, with five dietary digestible lysine levels $(1.06,1.12,1.18,1.24$, and $1.30 \%)$ and two sexes (males and females), totaling ten treatments with eight replicates of 22 birds each. From one to 10 days of age, birds were fed a commercial feed based on corn and soybean meal formulated according to the nutritional recommendations of Rostagno et al. (2005).
A basal feed was manufactured with $22.0 \%$ crude protein, 3,000 kcal ME/kg, and $1.06 \%$ digestible lysine to which increasing lysine $\mathrm{HCl}$ levels $(0.06 \%)$ were added, maintaining minimum digestible amino acid/ digestible lysine ratios of $72 \%$ Met+Cys, $65 \%$ Thr, $18 \%$ Try, $75 \%$ Val, and $105 \%$ Arg in all treatments. The experimental diets were obtained diluting a highlysine diet $(1.30 \%$ dig. Lys) with a low-lysine diet (1.06\% dig. Lys), as shown in Tables 1 and 2.

Table 1 - High (HLD) and low lysine (LLD) diets used during the starter phase (10-21 days).

\begin{tabular}{|c|c|c|}
\hline & \multicolumn{2}{|c|}{ Starter phase } \\
\hline & LLD & HLD \\
\hline Corn & 35.936 & 35.936 \\
\hline Soybean meal $45 \%$ & 33.958 & 33.958 \\
\hline Low-tannin sorghum & 20.000 & 20.000 \\
\hline Corn gluten $60 \%$ & 3.000 & 3.000 \\
\hline Oil & 2.523 & 2.523 \\
\hline Dicalcium phosphate & 1.780 & 1.780 \\
\hline Limestone & 0.893 & 0.893 \\
\hline Salt & 0.484 & 0.484 \\
\hline Starch & 0.892 & 0.100 \\
\hline DL-Methionine 99\% & 0.146 & 0.321 \\
\hline L-Lysine $\mathrm{HCl} 79 \%$ & 0.073 & 0.377 \\
\hline L-threonine $98 \%$ & -- & 0.113 \\
\hline L-arginine 99\% & -- & 0.031 \\
\hline L-valine 99\% & -- & 0.028 \\
\hline Glycine 99\% & -- & 0.141 \\
\hline Choline chloride $60 \%$ & 0.100 & 0.100 \\
\hline Vitamin suppl. ${ }^{1}$ & 0.100 & 0.100 \\
\hline Mineral suppl. ${ }^{2}$ & 0.050 & 0.050 \\
\hline Salinomycin $12 \%$ & 0.055 & 0.055 \\
\hline Antioxidant & 0.010 & 0.010 \\
\hline Total & 100.000 & 100.000 \\
\hline \multicolumn{3}{|l|}{ Calculated composition } \\
\hline ME (kcal/kg) & 3.000 & 3.000 \\
\hline $\mathrm{CP}(\%)$ & 22.00 & 22.00 \\
\hline Calcium (\%) & 0.884 & 0.884 \\
\hline Avail. phosphorus (\%) & 0.442 & 0.442 \\
\hline Sodium (\%) & 0.214 & 0.214 \\
\hline Potassium (\%) & 0.802 & 0.802 \\
\hline Dig. Lysine (\%) & 1.060 & 1.300 \\
\hline Dig. met+cys (\%) & 0.763 & 0.936 \\
\hline Dig. threonine (\%) & 0.740 & 0.845 \\
\hline Dig. tryptophan & 0.236 & 0.236 \\
\hline Dig. arginine (\%) & 1.334 & 1.365 \\
\hline Dig. valine (\%) & 0.947 & 0.975 \\
\hline
\end{tabular}

1 Vitamin supplement (Rovimix, Roche)- Guaranteed levels per kg product: Vitamin A 10,000,000 IU; Vitamin D3 - 2,000,000 IU; Vitamin E - 30,000 IU; Vitamin B1 - 2.0g ;Vitamin B6 - $4.0 \mathrm{~g}$; pantothenic acid - 12.0g; biotin- $0.10 \mathrm{~g}$; Vitamin K3 - $3.0 \mathrm{~g}$; folic acid - $1.0 \mathrm{~g}$; nicotinic acid- $50.0 \mathrm{~g}$; Vitamin B12 $-15.000 \mathrm{mcg}$; selenium- $0.25 \mathrm{~g}$; excipient q.s.p. 1,000g

${ }^{2}$ Mineral supplement (Roligomix, Roche)- Guaranteed levels per kg product: manganese $16.0 \mathrm{~g}$; iron - $100.0 \mathrm{~g}$; zinc $-100.0 \mathrm{~g}$; copper $-20.0 \mathrm{~g}$; cobalt - $2.0 \mathrm{~g}$; iodine -2.0 g; excipient q. s. p. $-1,000 \mathrm{~g}$. 
Table 2 - Dilution diagram and amino acid levels of the experimental diets ${ }^{1}$

\begin{tabular}{|c|c|c|c|c|c|c|c|}
\hline \multirow[t]{2}{*}{ Treatment } & \multicolumn{2}{|c|}{ Ratio (\%) } & \multirow{2}{*}{$\begin{array}{l}\text { Dig. } \\
\text { lysine } \\
(\%)\end{array}$} & \multirow{2}{*}{$\begin{array}{c}\text { Dig. } \\
\text { met+cys } \\
(\%)\end{array}$} & \multirow{2}{*}{$\begin{array}{l}\text { Dig. } \\
\text { threonine } \\
(\%)\end{array}$} & \multirow{2}{*}{$\begin{array}{l}\text { Dig. } \\
\text { arginine } \\
(\%)\end{array}$} & \multirow{2}{*}{$\begin{array}{c}\text { Dig. } \\
\text { valine } \\
(\%)\end{array}$} \\
\hline & LLD $^{1}$ & $\mathrm{HLD}^{1}$ & & & & & \\
\hline T1 & 100 & 0 & 1.060 & 0.763 & 0.740 & 1.334 & 0.947 \\
\hline $\mathrm{T} 2$ & 75 & 25 & 1.120 & 0.806 & 0.766 & 1.342 & 0.954 \\
\hline T3 & 50 & 50 & 1.180 & 0.849 & 0.793 & 1.350 & 0.961 \\
\hline T4 & 25 & 75 & 1.240 & 0.893 & 0.819 & 1.357 & 0.968 \\
\hline T5 & 0 & 100 & 1.300 & 0.936 & 0.845 & 1.365 & 0.975 \\
\hline
\end{tabular}

'LLD - low lysine diet; HLD - high lysine diet

Average minimum and maximum temperatures measured during the experimental period were 20.4 and $27.5^{\circ} \mathrm{C}$, respectively. At the end of the experimental period, birds and feed residues were weighed to subsequently calculate weight gain, feed intake, and feed conversion ratio. Mortality was recorded during the experiment and taken into account to correct performance data.

In the second experiment, 1600 male and female Cobb 500 broilers were used. A randomized block experimental design in a $5 \times 2$ factorial arrangement was applied, with five dietary digestible lysine levels $(0.92,0.98,1.04,1.10$, and $1.16 \%)$ and two sexes (males and females), totaling ten treatments with eight replicates of 20 birds each. From one to 21 days of age (pre-experimental periods), birds were fed a commercial feed based on corn and soybean meal formulated according to the nutritional recommendations of Rostagno et al. (2005).

A basal feed was manufactured with $20.0 \%$ crude protein, 3,100 kcal ME/kg, and $0.92 \%$ digestible lysine to which increasing lysine $\mathrm{HCl}$ levels $(0.06 \%)$ were added, maintaining minimum digestible amino acid/digestible lysine ratios of $73 \%$ Met+Cys, $65 \%$ Thr, 18\% Try, 77\% Val, and 105\% Arg in all treatments. The experimental diets were obtained by diluting a low-lysine diet $(0.92 \%$ dig. Lys) with a high-lysine diet (1.16\% dig. Lys), as shown in Tables 3 and 4.

Average minimum and maximum temperatures measured during the experimental period were 20.5 and $26.8{ }^{\circ} \mathrm{C}$, respectively. At the end of the experimental period, birds and feed residues were weighed to subsequently calculate weight gain, feed intake, and feed conversion ratio.
Table 3 - High (HLD) and low lysine (LLD) diets used during the grower phase (22-35 days).

\begin{tabular}{|c|c|c|}
\hline & \multicolumn{2}{|c|}{ Grower phase } \\
\hline & LLD & HLD \\
\hline Corn & 41.070 & 41.070 \\
\hline Soybean meal 45\% & 28.609 & 28.609 \\
\hline Low-tannin sorghum & 20.000 & 20.000 \\
\hline Corn gluten $60 \%$ & 3.000 & 3.000 \\
\hline Oil & 3.154 & 3.154 \\
\hline Dicalcium phosphate & 1.642 & 1.642 \\
\hline Limestone & 0.855 & 0.855 \\
\hline Salt & 0.461 & 0.461 \\
\hline Starch & 0.743 & 0.100 \\
\hline DL-methionine $99 \%$ & 0.097 & 0.274 \\
\hline L-Lysine $\mathrm{HCl} 79 \%$ & 0.054 & 0.359 \\
\hline L-threonine $98 \%$ & -- & 0.091 \\
\hline L-arginine 99\% & -- & 0.039 \\
\hline L-valine 99\% & -- & 0.032 \\
\hline Choline chloride $60 \%$ & 0.100 & 0.100 \\
\hline 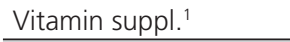 & 0.100 & 0.100 \\
\hline Mineral suppl. ${ }^{2}$ & 0.050 & 0.050 \\
\hline Salinomycin & 0.055 & 0.055 \\
\hline Antioxidant $^{3}$ & 0.010 & 0.010 \\
\hline Total & 100.00 & 100.00 \\
\hline \multicolumn{3}{|l|}{ Calculated composition } \\
\hline $\mathrm{ME}(\mathrm{kcal} / \mathrm{kg})$ & 3,100 & 3,100 \\
\hline $\mathrm{CP}(\%)$ & 20.000 & 20.000 \\
\hline Calcium (\%) & 0.824 & 0.824 \\
\hline Avail. phosphorus (\%) & 0.411 & 0.411 \\
\hline Sodium (\%) & 0.205 & 0.205 \\
\hline Potassium (\%) & 0.720 & 0.720 \\
\hline Dig. Lysine (\%) & 0.920 & 1.160 \\
\hline Dig. met+cys (\%) & 0.672 & 0.847 \\
\hline Dig. threonine (\%) & 0.670 & 0.754 \\
\hline Dig. tryptophan (\%) & 0.209 & 0.209 \\
\hline Dig. arginine (\%) & 1.182 & 1.220 \\
\hline Dig. valine (\%) & 0.861 & 0.893 \\
\hline
\end{tabular}

'Rovimix (Roche)- Guaranteed levels per kg product: vit A - 10,000,000 IU; vit D3 2,000,000 IU; Vit E - 30,000 IU; Vit B1 - 2.0g; vit B6 - 4.0 g; pantothenic acid - 12.0g; biotin- $0.10 \mathrm{~g}$; Vit K3 - $3.0 \mathrm{~g}$; folic acid - $1.0 \mathrm{~g}$; nicotinic acid - $50.0 \mathrm{~g}$; Vit B12 - 15.000 $\mathrm{mcg}$; selenium- $0.25 \mathrm{~g}$; and excipient q. s. $\mathrm{p}-1,000 \mathrm{~g}$

${ }^{2}$ Roligomix (Roche)- Guaranteed levels per kg product: manganese $16.0 \mathrm{~g}$; iron $100.0 \mathrm{~g}$; zinc $-100.0 \mathrm{~g}$; copper $-20.0 \mathrm{~g}$; cobalt $-2.0 \mathrm{~g}$; iodine $-2.0 \mathrm{~g}$; excipient q. s. p. $-1,000 \mathrm{~g}$.

${ }^{3}$ BHT (Beta hydroxybutyl toluene).

Table 4 - Dilution diagram and amino acid levels of the experimental diets ${ }^{1}$

\begin{tabular}{cccccccc}
\hline Treatment & \multicolumn{2}{c}{ Ratio (\%) } & \begin{tabular}{c} 
Dig. \\
lysine \\
\cline { 3 - 6 }
\end{tabular} & $\begin{array}{c}\text { Dig. } \\
\text { met+cys } \\
(\%)\end{array}$ & $\begin{array}{c}\text { Dig. } \\
\text { threonine } \\
(\%)\end{array}$ & $\begin{array}{c}\text { Dig. } \\
\text { arginine } \\
(\%)\end{array}$ & $\begin{array}{c}\text { Dig. } \\
\text { valine } \\
(\%)\end{array}$ \\
\hline T1 & 100 & 0 & 0.920 & 0.672 & 0.670 & 1.182 & 0.861 \\
\hline T2 & 75 & 25 & 0.980 & 0.715 & 0.691 & 1.192 & 0.869 \\
\hline T3 & 50 & 50 & 1.040 & 0.759 & 0.712 & 1.201 & 0.877 \\
\hline T4 & 25 & 75 & 1.100 & 0.803 & 0.733 & 1.211 & 0.885 \\
\hline T5 & 0 & 100 & 1.160 & 0.847 & 0.754 & 1.220 & 0.893 \\
\hline
\end{tabular}

'LLD - low lysine diet; HLD - high lysine diet 
At the end of the experiment and after approximately eight hours of fasting to empty the digestive tract, three birds per experimental unit, with the average body weight of the replicate, were sacrificed by neck dislocation to determine carcass weight and yield, and parts absolute weight and yield (breast with bone and skin, breast fillet, thigh, and drumstick), and abdominal fat. Carcass yield was calculated relative to live weight at slaughter, and parts and abdominal fat yields were determined relative to empty carcass weight.

The results obtained in both experiments were statistically analyzed using the statistical package Sistema de Análises Estatísticas e Genéticas (SAEG, 2000) developed by the Federal University of Viçosa (UFV). Lysine requirement estimates were determined using linear and quadratic regression models. When interactions were significant, lysine levels were nested within sex. Requirements were subsequently estimated by linear, quadratic and/or linear response plateau (LRP) equations.

\section{RESULTS AND DISCUSSION}

There was a quadratic effect $(p<0.05)$ of lysine levels on weight gain and feed conversion ratio of both males and females (Table 5). Digestible lysine requirements were higher for females than for males, differently from the recommendations of Rostagno et al. (2011).

Table 5 - Effect of digestible lysine level on the performance of male and female broilers in the starter phase (10-21 days of age).

\begin{tabular}{ccccccc}
\hline \multirow{2}{*}{$\begin{array}{c}\text { Dig. lysine } \\
\text { level (\%) }\end{array}$} & \multicolumn{2}{c}{ Weight gain (g) } & \multicolumn{2}{c}{ Feed intake $(\mathrm{g})$} & \multicolumn{2}{c}{$\begin{array}{c}\text { Feed conversion } \\
\text { ratio }\end{array}$} \\
\cline { 2 - 7 } & Males & Females & Males & Females & Males & Females \\
\hline 1.06 & 579 & 522 & 856 & 785 & 1.479 & 1.504 \\
\hline 1.12 & 583 & 527 & 857 & 775 & 1.469 & 1.470 \\
\hline 1.18 & 589 & 533 & 842 & 780 & 1.431 & 1.464 \\
\hline 1.24 & 575 & 538 & 839 & 787 & 1.458 & 1.465 \\
\hline 1.30 & 581 & 530 & 839 & 775 & 1.450 & 1.461 \\
\hline Mean & $581.7^{\text {a }}$ & $530.2^{\mathrm{b}}$ & $846.7^{\mathrm{a}}$ & $780.4^{\mathrm{b}}$ & $1.457^{\mathrm{b}}$ & $1.473^{\mathrm{a}}$ \\
\hline Regression & $\mathrm{Q}^{1}$ & $\mathrm{Q}^{2}$ & $\mathrm{~L}^{3}$ & $\mathrm{~ns}^{4}$ & $\mathrm{Q}^{5}$ & $\mathrm{Q}^{6}$ \\
\hline $\begin{array}{c}\text { Requirement, } \\
\%\end{array}$ & 1.15 & 1.22 & 1.30 & 1.06 & 1.22 & 1.24 \\
\hline \begin{tabular}{c} 
CV (\%) \\
\hline
\end{tabular} & 1.74 & & 1.81 & & 1.68 \\
\hline
\end{tabular}

$a, b$ Means followed by different letters in the same row are statistically different $(p<0.05)$.

${ }^{1}$ Quadratic effect: $Y=-138.865+1254.30 X-543.601 X^{2} ; R^{2}=0.62(p<0.05)$

${ }^{2}$ Quadratic effect: $Y=-233.032+1254.59 X-512.529 X^{2} ; R^{2}=0.88(p<0.05)$

${ }^{3}$ Linear effect $Y=973.234-108.454 X ; R^{2}=0.92(p<0.01)$.

${ }^{4}$ Not significant.

${ }^{5}$ Quadratic effect: $Y=3.4898-3.34516 X+1.36816 X^{2} ; R^{2}=0.60(p<0.05)$.

${ }^{6}$ Quadratic effect: $Y=3.50069-3.303 X+1.33543 X^{2} ; R^{2}=0.91(p<0.05)$.
Lysine levels also linearly affected $(p<0.01)$ male feed intake, indicating that feed intake decreased as dietary lysine levels increased; however, when lysine intake was calculated, there was a linear increase from $9.07 \mathrm{~g}$ to $10.91 \mathrm{~g}$ with dietary lysine levels of 1.06 to $1.30 \%$, respectively. Female feed intake was not significantly affected, consistent with the findings of Costa et al. (2001).

As previously observed by Han et al. (1991), Vasquez \& Pesti, (1997), and Bellaver et al. (1999), the regression model estimated higher requirements for feed conversion ratio compared with weight gain, both in males and females.

There was a quadratic effect $(p<0.01)$ of lysine levels on weight gain and feed conversion ratio of males in the grower phase (Table 6), corresponding to estimated digestible lysine requirements of 1.05 and $1.07 \%$, respectively. In females, there was a linear effect $(p<0.01)$ on weight gain and feed conversion ratio requirements, calculated in $1.16 \%$ digestible lysine.

Table 6 - Effect of digestible lysine level on the performance of male and female broilers in the grower phase (22-35 days of age).

\begin{tabular}{|c|c|c|c|c|c|c|}
\hline \multirow[t]{2}{*}{$\begin{array}{l}\text { Dig. lysine } \\
\text { level (\%) }\end{array}$} & \multicolumn{2}{|c|}{ Weight gain (g) } & \multicolumn{2}{|c|}{ Feed intake (g) } & \multicolumn{2}{|c|}{$\begin{array}{c}\text { Feed conversion } \\
\text { ratio }\end{array}$} \\
\hline & Males & Females & Males & Females & Males & Females \\
\hline 0.92 & 1191 & 994 & 2100 & 1802 & 1.771 & 1.814 \\
\hline 0.98 & 1210 & 1007 & 2077 & 1809 & 1.717 & 1.797 \\
\hline 1.04 & 1236 & 1016 & 2093 & 1813 & 1.694 & 1.785 \\
\hline 1.10 & 1197 & 1052 & 2065 & 1817 & 1.726 & 1.759 \\
\hline 1.16 & 1204 & 1039 & 2069 & 1826 & 1.721 & 1.758 \\
\hline Mean & $1207.6^{a}$ & $1021.7^{b}$ & $2081.2^{a}$ & 1813.7 & $1.726^{a}$ & $1.783^{b}$ \\
\hline Regression & $\mathrm{Q}^{1}$ & $L^{2}$ & $n s^{3}$ & ns & $\mathrm{Q}^{4}$ & $L^{5}$ \\
\hline $\begin{array}{c}\text { Requirement, } \\
\%\end{array}$ & 1.05 & 1.16 & 0.92 & 0.92 & 1.07 & 1.16 \\
\hline CV (\%) & \multicolumn{2}{|c|}{1.60} & \multicolumn{2}{|c|}{1.92} & \multicolumn{2}{|c|}{1.72} \\
\hline \multicolumn{7}{|c|}{$\begin{array}{l}\text { a,b Means followed by different letters in the same row are statistically different }(p<<\text { < } \\
{ }^{1} \text { Quadratic effect: } Y=-966.98+4171.83 X-1988.44 X^{2} ; R^{2}=0.54(p<0.01) \text {. } \\
{ }^{2} \text { Linear effect } Y=741.857+270.187 X ; R^{2}=0.95(p<0.01) \\
{ }^{3} \text { not significant. } \\
{ }^{4} \text { Quadratic effect: } Y=5.14394-6.46492 X+3.03593 X^{2} ; R^{2}=0.79(p<0.01) \\
{ }^{5} \text { Linear effect } Y=2.19203-0.402526 X ; R^{2}=0.96(p<0.01)\end{array}$} \\
\hline
\end{tabular}

There was no effect of digestible lysine levels on the feed intake of both sexes. According to Parr \& Summers (1991), in addition of energy, dietary amino acid imbalance influences feed intake. Therefore, it may be inferred that the maintenance of the ratio between lysine and the most critical amino acids for broilers (methionine, threonine, isoleucine, valine, and tryptophan) at the different evaluated lysine levels, as well as the fact the diets contained equal energy and protein levels, explains the similar feed intake among treatments obtained with both sexes. 
Carcass and parts weights (Tables 7 and 8) show that both in males and females there was a linear effect of lysine levels on breast weight $(p<0.01$ and $p<0.08)$, breast fillet weight $(p<0.02)$, and leg (thigh+drumstick) weight $(p<0.01$ and $p<0.08)$, corresponding to $1.16 \%$ digestible lysine requirement for each parameter. However, male leg weight linearly decreased as dietary digestible lysine level increased. Lysine levels did not significantly influence carcass weight of males or females, but there was a linear effect $(p<0.01)$ on female abdominal fat weight, with an estimated requirement of $1.16 \%$ digestible lysine.

Table 7 - Effect of digestible lysine level on carcass and parts weights of male and female broilers at 35 days of age.

\begin{tabular}{ccccccc}
\hline \multirow{2}{*}{$\begin{array}{c}\text { Dig. lysine } \\
\text { level }(\%)\end{array}$} & \multicolumn{2}{c}{ Carcass $(\mathrm{g})$} & \multicolumn{2}{c}{ Abdominal fat $(\mathrm{g})$} & \multicolumn{2}{c}{ Leg $(\mathrm{g})$} \\
\cline { 2 - 7 } & Males & Females & Males & Females & Males & Females \\
\hline 0.92 & 1366 & 1173 & 18.9 & 22.5 & 398 & 324 \\
\hline 0.98 & 1373 & 1194 & 18.8 & 19.4 & 388 & 333 \\
\hline 1.04 & 1370 & 1201 & 20.3 & 17.5 & 384 & 327 \\
\hline 1.10 & 1369 & 1200 & 18.1 & 18.3 & 391 & 336 \\
\hline 1.16 & 1376 & 1209 & 17.2 & 15.7 & 382 & 343 \\
\hline Mean & $1370.8^{\mathrm{a}}$ & $1195.4^{\mathrm{b}}$ & 18.7 & 18.7 & $388.4^{\mathrm{a}}$ & $332.6^{\mathrm{b}}$ \\
\hline Regression & $\mathrm{ns}^{1}$ & $\mathrm{~ns}$ & $\mathrm{~ns}$ & $\mathrm{~L}^{2}$ & $\mathrm{~L}^{3}$ & $\mathrm{~L}^{4}$ \\
\hline $\begin{array}{c}\text { Requirement, } \\
\%\end{array}$ & ---- & ---- & ---- & 1.16 & 1.16 & 1.16 \\
\hline CV (\%) & \multicolumn{2}{c}{3.83} & \multicolumn{2}{c}{23.35} & \multicolumn{2}{c}{4.00} \\
\hline
\end{tabular}

a,b Means followed by different letters in the same row are statistically different $(p<0.05)$. ${ }^{1}$ Not significant.

2 Linear effect: $Y=43.9653-24.3056 X ; R^{2}=0.84(p<0.01)$.

${ }^{3}$ Linear effect: $Y=437.549-47.222 X ; R^{2}=0.52(p<0.08)$

${ }^{4}$ Linear effect: $Y=262.889+67.0139 X ; R^{2}=0.50(p<0.02)$.

Table 8 - Effect of digestible lysine level on carcass parts weight of male and female broilers at 35 days of age.

\begin{tabular}{ccccc}
\hline \multirow{2}{*}{$\begin{array}{c}\text { Dig. Iysine level } \\
(\%)\end{array}$} & \multicolumn{2}{c}{ Breast $(\mathrm{g})$} & \multicolumn{2}{c}{ Breast fillet $(\mathrm{g})$} \\
\cline { 2 - 5 } & Males & Females & Males & Females \\
\hline 0.92 & 435.8 & 388.8 & 324.0 & 277.7 \\
\hline 0.98 & 445.0 & 392.4 & 332.3 & 289.1 \\
\hline 1.04 & 448.0 & 399.0 & 328.1 & 296.4 \\
\hline 1.10 & 456.7 & 399.6 & 335.6 & 299.2 \\
\hline 1.16 & 465.5 & 405.3 & 351.5 & 300.6 \\
\hline Mean & 450.2 & 397.0 & 334.3 & 292.6 \\
\hline Regression & $\mathrm{L}^{1}$ & $\mathrm{~L}^{2}$ & $\mathrm{~L}^{3}$ & $\mathrm{~L}^{4}$ \\
\hline Requirement, \% & 1.16 & 1.16 & 1.16 & 1.16 \\
\hline CV (\%) & \multicolumn{4}{c}{6.40} \\
\hline
\end{tabular}

Means followed by different letters in the same row are statistically different $(p<0.05)$. 1 Linear effect: $Y=327.069+118.403 X ; R^{2}=0.98(p<0.01)$.

${ }^{2}$ Linear effect: $Y=327.125+67.1875 X ; R^{2}=0.96(p<0.08)$.

3 Linear effect: $Y=233.181+97.222 X ; R^{2}=0.76(p<0.02)$

${ }^{4}$ Linear effect: $Y=195.637+93.229 X ; R^{2}=0.88(p<0.02)$.

Relative to carcass and parts yield (Tables 9 and 10), there was a linear effect of lysine level on breast fillet yield both in males and females $(p<0.01$ and $p<0.03)$. Breast yield and leg yield were significantly $(p<0.01)$ affected only in males, whereas for abdominal fat yield, consistent with the effect on abdominal fat weight, only females presented a linear response.
Table 9 - Effect of digestible lysine level on carcass and parts yield of male and female broilers at 35 days of age.

\begin{tabular}{ccccccc}
\hline \multirow{2}{*}{$\begin{array}{c}\text { Dig. lysine level } \\
(\%)\end{array}$} & \multicolumn{2}{c}{ Carcass (\%) } & \multicolumn{3}{c}{ Abdominal fat (\%) } & \multicolumn{2}{c}{ Leg (\%) } \\
\cline { 2 - 7 } & Males & Females & Males & Females & Males & Females \\
\hline 0.92 & 71.1 & 70.3 & 1.33 & 1.91 & 29.1 & 27.6 \\
\hline 0.98 & 71.4 & 71.0 & 1.37 & 1.62 & 28.3 & 28.0 \\
\hline 1.04 & 70.5 & 71.5 & 1.47 & 1.45 & 28.0 & 27.2 \\
\hline 1.10 & 71.2 & 70.7 & 1.32 & 1.52 & 28.6 & 28.0 \\
\hline 1.16 & 70.4 & 70.9 & 1.24 & 1.39 & 27.8 & 28.2 \\
\hline Mean & 70.9 & 70.9 & $1.34^{\mathrm{b}}$ & $1.58^{\mathrm{a}}$ & $28.3^{\mathrm{a}}$ & $27.8^{\mathrm{b}}$ \\
\hline Regression & $\mathrm{ns} \mathbf{n}^{1}$ & $\mathrm{~ns}$ & $\mathrm{~ns}$ & $\mathrm{~L}^{2}$ & $\mathrm{~L}^{3}$ & $\mathrm{~ns}$ \\
\hline Requirement, \% & ---- & ---- & ---- & 1.16 & 1.16 & ---- \\
\hline CV (\%) & \multicolumn{2}{c}{2.29} & \multicolumn{2}{c}{22.38} & \multicolumn{2}{c}{3.13} \\
\hline
\end{tabular}

Means followed by different letters in the same row are statistically different $(p<0.05)$. ${ }^{1}$ Not significant:

${ }^{2}$ Linear effect: $Y=3.5675-1.9113 X ; R^{2}=0.84(p<0.01)$

${ }^{3}$ Linear effect: $Y=32.434-3.9318 X ; R^{2}=0.54(p<0.02)$.

Table 10 - Effect of digestible lysine level on parts yield of male and female broilers at 35 days of age.

\begin{tabular}{|c|c|c|c|c|}
\hline \multirow{2}{*}{$\begin{array}{l}\text { Dig. lysine } \\
\text { level (\%) }\end{array}$} & \multicolumn{2}{|c|}{ Breast (\%) } & \multicolumn{2}{|c|}{ Breast fillet (\%) } \\
\hline & Males & Females & Males & Females \\
\hline 0.92 & 32.2 & 33.1 & 23.8 & 23.7 \\
\hline 0.98 & 32.4 & 32.7 & 24.2 & 23.8 \\
\hline 1.04 & 32.8 & 33.2 & 24.0 & 24.7 \\
\hline 1.10 & 33.4 & 33.3 & 24.5 & 24.9 \\
\hline 1.16 & 33.8 & 33.4 & 25.6 & 24.8 \\
\hline Mean & 32.9 & 33.2 & 24.4 & 24.4 \\
\hline Regression & $L^{1}$ & $n s^{2}$ & $L^{3}$ & $L^{4}$ \\
\hline $\begin{array}{c}\text { Requirement, } \\
\%\end{array}$ & 1.16 & ---- & 1.16 & 1.16 \\
\hline CV (\%) & \multicolumn{2}{|c|}{3.16} & \multicolumn{2}{|c|}{4.82} \\
\hline $\begin{array}{l}{ }^{1} \text { Linear effect: } Y= \\
{ }^{2} \text { Not significant. } \\
{ }^{3} \text { Linear effect: } Y= \\
{ }^{4} \text { Linear effect: } Y=\end{array}$ & $\begin{array}{l}608+6 \\
753+5\end{array}$ & $\begin{array}{l}; R^{2}=0.99 \\
; R^{2}=0.76 \\
R^{2}=0.85\end{array}$ & $\begin{array}{l}\text { 01). } \\
\text { 1). } \\
\text { 3). }\end{array}$ & \\
\hline
\end{tabular}

The digestible lysine requirement level of $1.16 \%$ determined for breast and breast fillet yields is higher than that estimated for maximum weight gain $(1.05 \%)$ and feed conversion ratio $(1.07 \%)$, indicating higher digestible lysine requirements to maximize breast and breast fillet yields. This demonstrates that there is a hierarchy in lysine requirements, as previously suggested by Leclercq (1998b). Differently from the results of the present study, the Cobb manual (2003) indicates $1.02 \%$ digestible lysine for "maximum white meat yield", which is lower than the proposed level of $1.04 \%$ digestible lysine for maximum growth rate and feed conversion ratio improvement. 


\section{CONCLUSIONS}

Digestible lysine requirements for maximum performance of Cobb 500 broilers are $1.22 \%$ for males and $1.24 \%$ for females in the starter phase (10 to 21 days of age) and $1.16 \%$ for both sexes in the grower phase (22 to 35 days of age).

\section{REFERENCES}

Bellaver C, Brum PAR, Guidoni AL, Scheuermann GN, Rosa PS, Snizek P. Estimativas das exigências de energia e lisina metabolizáveis em frangos de corte de 1 a 21 dias de idade. Anais da Reunião Anual da Sociedade Brasileira de Zootecnia; 1999; Porto Alegre, Rio Grande do Sul. Brasil.

Cobb. Cobb broiler nutrition guide. The Cobb Vantress Company; 2003.

Costa FGP, Rostagno HS, Albino LFT, Gomes PC, Toledo RS. Níveis dietéticos de lisina para frangos de corte nos períodos de 01 a 21 e 22 a 42 dias de idade. Revista Brasileira de Zootecnia 2001;30(5):1490-1497.

Grisoni ML. Role des acides aminés alimentaires dans la lipogenese du poulet de chair [thesis]. Paris (FR): University of Aix-Marseille;1991.

Han Y, Baker DH. Lysine requirements of fast- and slow-growing broiler chicks. Poultry Science 1991;73:1739-1745.
Han Y, Baker DH. Digestible lysine requirement of male and female broiler chicks during the period tree to six weeks post hatching. Poultry Science 1994;73:1739-1745.

Leclerca B. El concepto de proteina ideal y el uso de aminoácidos sinteticos estudio comparativo entre pollos y cerdos. $14^{\circ}$ Curso de Especialización FEDNA: avances en nutrición y alimentación animal;1998b; Barcelona. Spain. p.191-202

Leclercq B. Lysine: specific effects of lysine on broiler production: comparison with threonine and valine. Poultry Science 1998a;77:118-123.

Parr JF, Summers JD. The effect of minimizing amino acid excesses in broiler diets. Poultry Science 1991;70:1540-W9

Rostagno HS, Albino LFT, Donzele JL, Gomes PC, Oliveira RF, Lopes DC, et al. Tabelas Brasileiras para Aves e Suínos - Composição de alimentos e exigências nutricionais. $2^{a}$ ed. Viçosa, MG: UFV; 2005. 186p.

Rostagno HS, Albino LFT, Donzele JL, Gomes PC, Oliveira RF, Lopes DC, et al. Tabelas brasileiras para aves e suínos - composição de alimentos e exigências nutricionais. $3^{a}$ ed. Viçosa, MG: UFV; 2011. 252p.

Sistema para Análise Estatísticas e Genéticas. versão 8.0. Viçosa: Fundação Arthur Bernardes; 2000

Vazquez M, Pesti GM. Estimation of the lysine requirement of broiler chicks for maximum body gain and feed efficiency. Journal Applied Poultry Research 1997:6:241-246. 